SCIO. Revista de Filosofia, n. ${ }^{\circ}$ 19, Noviembre de 2020, 209-228, ISSN: 1887-9853

\title{
ESTELA INDETERMINISTA DEL QUOD EST: FUNCIÓN DE ONDA Y POSIBILIDAD ESENCIAL
}

\author{
INDETERMINISTIC TRAIL OF QUOD EST: \\ WAVE FUNCTION AND ESSENTIAL POSSIBILITY
}

Vicente Llamas Roiga*

Fechas de recepción y aceptación: 28 de abril de 2020 y 24 de julio de 2020

DOI: $10.46583 /$ scio_2020.19.620

Resumen: La metafísica dinamicista que despunta en la segunda mitad del siglo XIII reivindicando el estatuto intencional o real disminuido de la esencia como tal a la luz de la novedosa lógica modal abre la senda al indeterminismo, con la sintaxis de mundos alternos suspendida en la radical contingencia de la creación. El ensayo plantea un diálogo de esa metafísica, en clave de ontología cuántica, con la moderna mecánica de estados posibles que continuará con la revisión del principio de identitas indiscernibilium sobre el carácter simétrico o antisimétrico de la función de onda.

Palabras clave: esencia, indeterminismo, identidad, fin, acto.

Abstract: The metaphysics of dynamism that emerges in the second half of the $13^{\text {th }}$ century -claiming the intentional or real status diminished from the essence as such in the light of the novel modal logicpaves the way to indeterminism, with the syntax of alternate worlds

\footnotetext{
${ }^{a}$ Dpto. Filosofía y Sociedad Pontificia. Universidad Antonianum (Murcia). Código ORCID: 00000003-4830-3003

${ }^{*}$ Correspondencia: Instituto Teológico de Murcia. Plaza Beato Andrés Hibernón, 3. 30001 Murcia. España.

E-mail: v.llamasroig@um.es
} 
dangling on the radical contingency of creation. The essay brings up a dialogue between that metaphysics, in a quantum ontological tone, and the modern mechanics of possible states which culminates in reviewing the principle of identitas indiscernibilium according to the symmetric or antisymmetric nature of the wave function.

Keywords: essence, indeterminism, identity, aim, act.

\section{§1. INTRODUCCIÓN: DIEDRO METAFÍSICO}

Hay una metafísica sedentaria del quo est, hipotecada en el acto, una directriz energetista de subrogación de la potencia como principio constitutivamente relativo al acto, fundamento ontológico de entidad y actividad. Bajo el visado predicamental del acto como forma, la materia (potencia en este orden de valoración) debe a aquel, a título de forma essendi (simpliciter), toda actualidad. En el orden transcendental en que el acto es licitado como esse, el actus essendi reporta a la esencia (con valor de potencia en esa inspección) la existencia singular ${ }^{1}$. La potencia se distingue realmente del acto en el complejo creado como respectividad a él, es real en cuanto tal (respectus realis ad actum). La distinción real de acto y potencia en el orden transcendental tiene raíz teológica, pende sobre el hiato entre una causalidad ejemplar divina de semblanza intelectual (esencia) y una causalidad eficiente adscrita a la esfera volitiva (esse), reclamando, además, una solución analógica dual, de atribución intrínseca, apta para la esencia, y de proporcionalidad, adecuada al esse.

En una línea metafísica disidente que prima ontológicamente la potencia sobre el acto (prius tempore et natura), el carácter adventicio o accidental del respectus preexige extremos absolutos, la entidad del extremo a quo para el cual el respectus es accidens relativum (privado de la subjetualidad a quo del accidente absoluto, pues ens ad alterum est sua accidentalitas ad fundamentum: accidentalitas accidentis entis ad alterum es el mismo accidens

\footnotetext{
1 "Que el acto es antes que la potencia significa que el ser de la potencia o es derivado del acto, o es simplemente no ser [...] Si la definición primera de potencia es «principio de cambio que está en otro o en el mismo en cuanto otro», ambos sentidos de la potencia comportarán la referencia a la alteridad: en un caso la novedad de lo otro irá en detrimento de sí mismo (y entonces será no ser), y en el segundo caso la novedad de lo otro no implicará pérdida de sí" (De Garay, 1987: 110-111).
} 
relativum, evitando una progresión superflua de entidades). Así, la potencia no es ya nimia respectus ad actum y cobra la consistencia de fundamento real para ese respectus, fuere extrinsecus o intrinsecus adveniens. Su realidad es, obviamente, de textura diversa a la del acto, una realidad disminuida. Se alza entonces la esencia en ens extra animam secundum quid o esse aliquid en el que «aliquid» importa una aliquitas nominal de res, a reor reris, y «esse» un ser simpliciter de res rata (ser extrapsíquico menor, ens diminutum o difusamente intencional por dotado del esse essentiae que la ratifica como existible, avalando su creaturalidad).

En la metafísica existencialista que encumbra al acto, este es principio transcendental de identidad, aun como forma sustancial. La tesis tomista de la distinción real y unidad transcendental de esencia y esse profundiza en la articulación metafísica de la identidad y la diferencia, señalando a la potencia como factor de aliedad y de abaliedad (conjugadas las cláusulas propter aliud esse / propter quod aliud est que ponen en relieve la relación causal) por su genética incapacidad de autoactualización: la prerrogativa onto-cronológica del acto transluce la preferencia de la identidad sobre la diferencia (potencia como razón de alteridad). Frente a ella, la metafísica esencialista de Duns Escoto transpone el factor de abaliedad al acto formal (no autoactualizante, acto recibido de otro: lo que un ente tiene ab alio, aquello en que deviene a expensas de una fuerza causal externa, dicta su potencia al acto formal; solo lo que posee per se y desde sí mismo lo acredita en acto virtual, su inherente dinamismo, su potencial autonomía activa, las capacidades de autodesarrollo por conjunción de actos formal y virtual en el mismo sujeto en tanto que actos de dispar especie).

La potencia virtual de ser antepone la diferencia a la identidad, los modos virtuales intrínsecos de singularización de la esencia a la comunidad realeidética que dispensa la natura communis o a la generalidad del unívoco ens, una primitas virtualitatis que imputa al individuo en su potencial existencia como prius ontológico (la individualidad es exponente ontológico último de incomunicabilidad ut quod para toda naturaleza común). El agente precontiene en su virtud activa posibilidades de autoactualización, y el estatuto de la forma a priori, al margen de la materia, es precisamente el de un efecto precontenido virtualmente en el poder eficiente. La potencia virtual no se opone 
al acto virtual, solapa con él como poder de autoefectuación ${ }^{2}$. El giro dinamicista estiliza la imagen de Dios, ya no tanto ipsum esse subsistens, acto puro en el que se funda su omnipotencia (el lema «agere sequitur esse» escolia al acto de ser como fundamento ontológico de toda acción: el poder de Dios y lo posible respectivo a él no tendrían soporte en sí mismos sino en el ser divino), cuanto potentia (absoluta / ordinata) o potestad. La vía del quo est relega la ejemplaridad al pobre papel que la esencia tiene en el complejo onto-eidético como modus assimilandi (el esse creaturae es actus assimilandi, assimilatio unilateral de lo creado al creador). La innovadora metafísica del quod est en la que la potencia deja de ser simple correlación con el acto, por contra, realza la causalidad ejemplar, desplazada al feudo volitivo divino (motio contingens ad intra) o al práctico-intelectivo, una apología de la esencia como término real de esa causalidad en su misma expectativa existencial o bajo licencia de existible.

La mecánica cuántica opera una reducción eidética de la física a escala microscópica en la estela de una lógica modal que agrega al modus ponens proposicional una novedosa regla inferencial de necesitación $n^{3}$. La metafísica vestigial de Duns Escoto o la ratitudinal de Enrique de Gante, su inequívoca vocación de salto al plano quiditativo o intencional, el de la esencia como potentia essendi en su diminuta entidad extrapsíquica (realitas secundum quid) o asistida por la prima intención que representa el esse simpliciter essentiae, auspician un tratamiento de la realidad que trazará la senda por la que dis-

\footnotetext{
2 "Con el concepto de actus virtualis Escoto tiene en cuenta que la potencia activa es más que la simple posibilidad de una determinada actividad. De hecho, en este caso la potencia en cuanto principio está in actu porque puede obrar en cualquier momento [...] no es lícito poner la potencia qua principio activo como opuesta al acto, como sí lo es, en cambio, poner a la potencia pasiva" (Hoeres, 1976: 341).

${ }^{3}$ En lógica modal, el símbolo primitivo de necesidad se deduce de la posibilidad por negación. La interdefinición de los símbolos de necesidad y posibilidad es sincrética a la de los cuantificadores en lógica de primer orden: $\exists x \phi(x)=n o \forall x$ no $\phi(x), \forall x \phi(x)=\nexists x$ no $\phi(x)$. La semántica modal maneja una tríada ordenada de elementos: conjunto de mundos posibles bajo relación de accesibilidad y una función que asigna valores de verdad a proposiciones del conjunto maximal-consistente de estas en que revierte cada mundo posible. Los mundos posibles juegan un papel crucial en la definición de operadores modales, no así en la de operadores lógicos no modales. La inaccesibilidad al resto de mundos posibles desde uno dado haría verdaderas todas las fórmulas necesitaristas de $\Phi$ en ese mundo aislado y falsas todas las posibles, pues las condiciones de verdad ligadas a la fórmula necesitarista no requieren la existencia de un mundo posible que sea accesible (la verdad de la necesidad de $\Phi$ en ese mundo posible solo exige la inaccesibilidad desde él de cualquier mundo en que $\Phi$ fuese falsa).
} 
curra, en su incipiente singladura, la mecánica indeterminista. La naturaleza ondulatoria de la materia difumina la especificidad local prevista por la física clásica en el orden corpuscular, y abre cauce solo a la probabilidad presencial. La medida de la posición de un gran número de sistemas idénticos, partículas con la misma función de onda unidimensional $\Psi(x)$, saldaría colecciones de valores correspondientes a distribuciones regionales de probabilidad con un valor medio (valor esperado). La probabilidad $\left[\psi^{2}(x) d x\right]$ local de una partícula en el elemento lineal $d x$ centrado en $x$ tendrá un valor esperado: $\langle x\rangle=\int_{-\infty}^{+\infty} x \psi^{2}(x) d x$.

Como la esencia devenga en su estatuto metafísico una posibilidad real de existencia singular en la línea eidética (existencia probable en una esencia ratificada como tal, provista de un esse essentiae, variedad menor de ser extra animam que positiva ontológicamente la realidad de la sola posibilidad existencial), la función cuadrática de onda mensura una densidad de probabilidad de presencia (presencia probable bajo un valor cuantizado de energía). La noción de acto virtual es cardinal en la visual cuántica: los valores propios de la función de onda son virtuales colapsos energéticos, virtuales enérgeias que definen el estado en que se manifiesta una partícula.

En el orden cuántico, cualquier propiedad funcionalizada $[f(x)]$ puede ser estimada probabilísticamente en su valor esperado: $\langle f(x)\rangle=\int_{-\infty}^{+\infty} f(x) \psi^{2}(x) d x$. La nueva visión mecánica de la realidad impone una ontología fenomenológica de observables y estados sistémicos con una incertidumbre normativa reductora de la precisión gnoseológica. En esa escena teórica, el principio de identitas indiscernibilium quedaría vinculado al solapamiento de funciones de onda (simétrica o antisimétrica).

Los diferentes valores propios de la función de onda o los de energía cuantizada conectan con la intrínseca modalización singular de la esencia en proyección existencial. La individualidad es modus intrinsecus essentiae, de suerte que la esencia se actualiza o se realiza ontológicamente cuantizada, modulada por diferencias últimas de cariz ontológico (haecceitas escotista) que no conceden por sí mismas la existencia, solo la posibilitan (la infinitud es un modo disyuntivo de la entidad como univocum quid o esencia más común: la metafísica univocista atenderá al «ente en cuanto quid unívoco», 
si ha de ser una ciencia de esencias, centrada en el ente antes de su modalización finita o infinita, y, a la vez, en la tensión ontológica por continencia virtual que esos modos generan ad intra en la entidad). La función de onda admite valores propios solo como virtuales singularizaciones sistémicas, y no es hasta el colapso bajo uno de esos eigenvalores que se deshace la cópula de posibilidades opuestas. También en la sutil metafísica del quod est, bajo la realidad individuada late la posibilidad de no ser amortizada en la indiferencia esencial de toda contingencia actual a la existencia (la esencia subyace en la neutralidad de su realidad metafísica e indiferencia a la existencia en cada modo singular de actualidad).

En la función de onda está, pues, probabilísticamente funcionalizado el eîdos de un sistema a escala microscópica, virtualmente individuado (átomo aislado) en valores propios. El enfoque cuántico armoniza con la sediciosa visión dinamicista de lo real que ofrece la metafísica del quod est, sustentada en la entidad extramental de la potencia que luce la esencia en cuanto tal. La semántica de mundos posibles es la expresión culminante de esa concepción epifenoménica de la forma en la creación frente al determinismo energético en la sagital tomista del quo est.

\section{§2. CuAntización DE LA ENERgía. El aCto INDividuAdo}

El estado de un sistema cuántico compendia todas sus características instantáneas. Los diversos estados son formalizados mediante vectores unitarios del espacio de Hilbert $(H)$, espacio vectorial complejo con un producto interno de ciertas propiedades, infinito número de vectores linealmente independientes, completo (espacio de Banach en el que toda sucesión convergente tiene un vector límite intrínseco) y separable (contiene un subconjunto infinito de vectores denso en $H$ ). Los estados cuánticos son combinables, rindiendo por superposición otro estado cuántico plegado a la ley de composición interna (suma vectorial) vigente en el espacio de Hilbert.

Dados el estado de un sistema y uno de los valores propios de un observable, puede sondearse la probabilidad de que la medida de dicho observable en el estado considerado arroje como resultado el valor propio en cuestión. 
Cada observable (propiedad mensurable) de un sistema cuántico se representa mediante un único operador hermítico (autoadjunto -solo admite conjuntos reales de valores propios- acotado de una transformación lineal del espacio de Hilbert), de modo que el dominio del operador englobe todos los valores posibles del observable. Los autovectores correspondientes al espectro (conjunto de eigenvalores) de cada observable definen una base en el espacio de Hilbert.

Los espacios de Hilbert en mecánica cuántica suelen ser de dimensión infinita, y en ellos, los operadores hermíticos no son necesariamente autoadjuntos, apartándose entonces del teorema de resolución espectral que rige para los hermíticos autoadjuntos de los espacios de dimensión finita. Los operadores no acotados (caso del hamiltoniano en ausencia de límite para el valor de la energía) no están habitualmente definidos en todo el espacio, solo en un domino denso de este (conjunto que intersecta a todo abierto no vacío del espacio, o equivalentemente, conjunto cuya adherencia es todo el espacio), catalogados como estados de energía infinita los no circunscritos a ese dominio. La compatibilidad de dos observables se basa en la conmutabilidad de sus hermíticos representativos. Los observables incompatibles (posición y momentum, por ejemplo, cuyos operadores respectivos no son conmutables) no pueden poseer estados propios simultáneos.

El producto de las desviaciones estándar de dos observables sobre el mismo estado está acotado (principio de indeterminación de Heisenberg: no es posible preparar colectivos de sistemas en el mismo estado cuántico para los que el producto de las dispersiones de las medidas de dos variables canónicas conjugadas sea inferior a cierto límite fenomenológico, no de sensibilidad de medida).

La diacronía sistémica, la evolución temporal de un estado, es descrita por la ecuación de Schrödinger, principal herramienta de que dispone esta mecánica para auditar funciones propias, una ecuación diferencial de segundo orden con explícita variación temporal del estado cuántico de un sistema sometido a un hamiltoniano especial que, en contraste al clásico, sumido en el espacio fásico del sistema, variedad simpléctica de dimensión finita, opera linealmente sobre elementos de un espacio de Hilbert: 


$$
\widehat{H}_{(\vec{r}, t)} \Psi(\vec{r}, t)=i \hbar \frac{\partial \Psi(\vec{r}, t)}{\partial t}
$$

Suele designarse hamiltoniano al operador energía total por analogía a la función correlativa en clásica:

$$
\widehat{H}_{(x, y, z, t)}=-\frac{\hbar^{2}}{2 m}\left(\frac{\partial^{2}}{\partial x^{2}}+\frac{\partial^{2}}{\partial y^{2}}+\frac{\partial^{2}}{\partial z^{2}}\right)+\widehat{U}_{(x, y, z, t)}=-\frac{\hbar^{2}}{2 m} \nabla_{(x, y, z)}^{2}+\widehat{U}_{(x, y, z, t)}
$$

$-\frac{\hbar^{2}}{2 m} \nabla_{(x, y, z)}^{2}$ es el operador energía cinética y $\widehat{U}_{(x, y, z, t)}$ el de energía potencial.

En la generalización tridimensional independiente del tiempo, la función $\Psi$ de onda y la energía potencial $U$ se expanden a las coordenadas cartesianas ordinarias. La forma de la solución es conjeturable por tratamiento probabilístico. En una caja tridimensional, la probabilidad de que una partícula se encontrase en un elemento de volumen $d x d y d z$ centrado en el punto $(x, y, z)$ sería: $\Psi^{2}(x, y, z) d x d y d z$, con $\Psi(x, y, z)$ como solución a la ecuación de onda reformateada ${ }^{4}$ :

$$
\psi(x, y, z)=A \operatorname{sen}\left(k_{1} x\right) \operatorname{sen}\left(k_{2} y\right) \operatorname{sen}\left(k_{3} z\right)
$$

La introducción de la solución en la ecuación proporciona valores cuantizados de energía $\left(E=\frac{\hbar^{2}}{2 m} \sum_{i=1}^{3} k_{i}^{2}\right)$ equivalentes a: $E=\frac{p_{x}^{2}+p_{y}^{2}+p_{z}^{2}}{2 m}$, subrepticio el aspecto corpuscular en las componentes cartesianas del momentum exhibidas por el trinomio.

${ }^{4}$ La función de onda $(\Psi)$ conjuga la doble naturaleza de lo fenoménico, ondulatoria y corpuscular, reflejando la amplitud de onda asociada a una partícula. No se trata de una magnitud física en el sentido convencional en que puedan serlo el momentum, la aceleración, etc. De hecho, puede adoptar la forma general de una función compleja, con parte imaginaria, de ahí que se maneje $|\Psi|^{2}=\Psi^{*} \Psi$, de manera que: $\Psi=\Psi^{*}$, si $\Psi$ fuese una función solo con parte real. El significado físico de la función está vinculado al de $|\Psi|^{2}$, que cuantifica la probabilidad de presencia de una partícula en una región determinada del espacio. Considerado un elemento de volumen $d V$ alrededor de cierto punto (x,y,z), la probabilidad de localización de la partícula en él sería: $P_{d V}=\left|\Psi^{2}(x, y, z)\right| d x d y d z$. Extensible por integración a un volumen finito en torno al punto genérico: $P_{V}=\int_{V}|\Psi|^{2} d V$.

$\Psi^{2}$ está normalizada en el espacio, extendida globalmente a él la integral anterior adopta valor unitario. La condición de normalización supone una drástica restricción del elenco de funciones matemáticas que puedan desempeñar el papel de $\Psi$. 
Para un átomo aislado, la energía potencial depende exclusivamente de la distancia radial electrón-centro del núcleo, de ahí la pertinente transformación directa de coordenadas rectangulares a esféricas $(r, \theta, \phi)$ en la ecuación de onda, con ulterior separación de variables:

$$
\psi(r, \theta, \phi)=R(r) \Theta(\theta) \Phi(\phi)
$$

La ecuación de Schrödinger se desglosa así en tres aplicaciones diferenciales correspondientes a cada una de las funciones esféricas presentadas, recluida la energía potencial en la ecuación radial, sin impacto de la forma de $U(r)$ sobre las angulares $\Theta(\theta)$ о $\Phi(\phi)$ :

$$
-\frac{\hbar^{2}}{2 m r^{2}}\left\{\frac{\partial}{\partial r}\left(r^{2} \frac{\partial \psi}{\partial r}\right)+\left[\frac{1}{\operatorname{sen} \theta} \frac{\partial}{\partial \theta}\left(\operatorname{sen} \theta \frac{\partial \psi}{\partial \theta}\right)+\frac{1}{\operatorname{sen}^{2} \theta} \frac{\partial^{2} \psi}{\partial \phi^{2}}\right]\right\}+U(r) \psi=E \psi
$$

$\operatorname{Con} U(r)=-k \frac{Z e^{2}}{r}$. Continuidad y normalización de la función de onda implementan un número cuántico asociado a cada una de sus tres dimensiones: principal $(n)$, orbital $(l)$ y magnético $\left(m_{l}\right)^{5}$.

${ }^{5}$ La función de distribución de probabilidad radial de un átomo con un solo electrón en estado fundamental sería: $A_{(1,0,0)}^{2} r^{2} e^{-\frac{2 Z r}{a_{0}}}$, y en el estado $n=2, l=1, m_{l}=0$, por ejemplo: $A^{\prime} \cos ^{2} \theta r^{4} e^{-\frac{Z r}{a_{0}}}$. Elemento de volumen en coordenadas esféricas: $d V=(r \operatorname{sen} \theta d \phi)(r d \theta) d r=r^{2} \operatorname{sen} \theta d \theta d \phi d r$.

La extensión de la integral que marca la normalización de la función de onda a todo el espacio:

$\int|\psi|^{2} d V=\int_{0}^{\infty}\left[\int_{0}^{\pi}\left(\int_{0}^{2 \pi}|\psi|^{2} r^{2} \operatorname{sen} \theta d \phi\right) d \theta\right] d r=1$.

Para el estado de mínima energía (fundamental): $\psi_{(1,0,0)}=A_{(1,0,0)} e^{-\frac{Z r}{a_{0}}}$, con lo que:

$\int|\psi|^{2} d V=\int_{0}^{\infty}\left[\int_{0}^{\pi}\left(\int_{0}^{2 \pi} A_{(1,0,0)}^{2} e^{-\frac{2 Z r}{a_{0}}} r^{2} \operatorname{sen} \theta d \phi\right) d \theta\right] d r=1$

Como $\Psi_{(1,0,0)}(r)$ es independiente de $\theta$ y $\Phi$, procede el craqueo de la integral triple en el producto de tres integrales:

$\int|\psi|^{2} d V=\left(\int_{0}^{2 \pi} d \phi\right)\left(\int_{0}^{\pi} \operatorname{sen} \theta d \theta\right)\left(\int_{0}^{\infty} A_{(1,0,0)}^{2} e^{-\frac{2 L_{1}}{a_{0}}} r^{2} d r\right)=2 \pi \cdot 2 \cdot A_{(1,0,0)}^{2}\left(\int_{0}^{\infty} e^{-\frac{2 Z r}{a_{0}}} r^{2} d r\right)=1$.

De la integración por partes: $\int_{0}^{\infty} r^{2} e^{-\frac{2 Z r}{a_{0}}} d r=\frac{a_{0}^{3}}{4 Z^{3}}$, resulta: $\psi_{(1,0,0)}=\frac{1}{\sqrt{\pi}}\left(\frac{Z}{a_{0}}\right)^{3 / 2} e^{-\frac{Z r}{a_{0}}}$.

La probabilidad de encontrar al electrón solitario en el intervalo $[r, r+d r]$ será: $|\psi|^{2} 4 \pi r^{2} d r$ (densidad de probabilidad radial: $|\psi|^{2} 4 \pi r^{2}$ ). 


\section{§3. VIA POTENTIAE: INDETERMINISMO Y COLAPSO SINGULAR}

La relevancia del acto en el modelo energetista concede protagonismo a la estructura, y en el orden accidental, la estructura materia-forma traduce la yuxtaposición cantidad-cualidad: la materia es principio sustancial de quantitas dimensiva y la forma lo sería de qualitas (el accidente quantitas y las propiedades cualitativas accidentales remiten a los co-principios esenciales del complejo sensible). El hilemorfo debe su unidad a la forma organizativa, principio de cohesión de partes constituyentes, y las posibilidades de perfeccionamiento de cada ente individuado están predeterminadas en una forma única (unius entis est una forma). No hay rígido estatismo, la estructura tiene cobertura dinámica energotélica, un régimen dinámico regido por formas de teleología no coyunturales ni restrictivamente intencionales, opuestas al azaroso devenir entrópico; predisposiciones, regularidades con tendencia predominante al equilibrio, interacciones conducentes con mayor frecuencia (causa ut in pluribus en el patrón estadístico de modalidades) (Pape, 1996) a la preservación y la optimización del orden natural que al deterioro o la desestabilización.

La metafísica finalista de Aristóteles y Tomás de Aquino aboga por una conservación de la energía que transluce ese vigor primario del acto: toda enérgeia tiene un télos (solidario a la supremacía axiomática del acto es el regio fuero del fin, no metafórico, real -si la acción presume un fundamento ontológico, el acto de ser, la prelación causal vendrá dictada por la preferencia ontológica; así, el imperio causal extrínseco del fin será una consecuencia de la prioridad ontológica de este: el fin último es prótos ón). Bajo efluvio de fines opacos, la eficiencia es una fuerza ciega, inepta, potestad huérfana suspendida en la potencia. El fin, en su actualidad primaria, mueve a la causa eficiente al acto. La inane eficiencia diatélica es desterrada, todo dinamismo se rige por una eficiencia teleológicamente dirigida, sin huecos ontológicos que saboteen la atenuada necesidad implícita en el ser donado dentro de los hemisferios del cosmos creatural (mórfico-forma subsistens / hilemórficoforma informans vel immanens). Solo per accidens, la interacción podría obedecer a ímpetus o descargas liberadoras (el clinamen es fortuito, ni siquiera responde al sesgo frecuencial causa ut in paucioribus, sino al hito ut in raro) 
(Faust, 1931; Knuuttila, 1978: 79-98), de ordinario refleja sistémicas tendencias isostáticas congruas con la homeostasia onto-eidética que estanca el atrio creatural en la íntima estructura de cada ente (esencia ordenada a un esse, no autosuficiente o autoconsistente como posibilidad pura a priori, y esse concebido, no como factum essendi, vacuo hecho de ser, sino como actus essendi, acto de una esencia). Los intercambios son niveladores o compensatorios, desvelando a la esencia en su vasta gama de tonalidades existenciales. En el orbe físico, el movimiento es la expresión de transiciones teleonómicas; en el biológico, la actividad orgánica encauza el curso vital al equilibrio interno, a la supervivencia, con eventuales malformaciones congénitas u ocasionales derivas genéticas, extravíos de la declinación natural debidos a obstructivas fuerzas morbosas, turbias corrientes patógenas que abortan posibilidades esenciales. El aislamiento es ficticio, los flujos de materia y energía presiden la dinámica natural, si bien la complejidad hilemórfica es morfo-télica, "y en atención al acto, morfo-energo-télica" (Polo, 1984: 210).

El veto al vacío energotélico es ilación de los principios de homeostasis onto-eidética y teleotropismo: ser y esencia solo se dan en conjunción real, con proclividad a la maximización energética, actualización teleotáxica de posibilidades de desarrollo codificadas genotípicamente en la esencia. La primacía inter- e intraentitativa del acto sobre la potencia en el orden transcendental de invocación de dichos principios (esse est prima rerum creatarum: ad intra, en la entidad finita, el esse prima sobre la esencia, como inter-entitativamente el esse subsistens lo hace sobre el insubsistente esse habens) modula la homeostasis en esa doble dimensión, e instaura una jerarquía analógica favorable al gradiente energético en la que lo más actual (formas subsistentes hiléfoba-anhilética y material) destaca sobre el plan hilemórfico, lo que convoca al ente a una interna progresión de actualidad por tropismo télico.

Dinamismos exergónicos o endergórnicos, inmanentes o transitivos, son trasunto de contingentes resistencias a la corrupción y de histéresis ontológica, reticencias a la deflación mórfica, a la pérdida de la forma actual, inclinación a la prevalencia de propiedades aun en ausencia del influjo causal estabilizante o frente a perturbadores estímulos, fuera de una teoría rupturista de catástrofes (propensión de sistemas hilemórficos estructuralmente estables a la discontinuidad o la divergencia). El determinismo que instruye la metafísica 
del fin fomenta una idea de histéresis contraria a la reversibilidad de procesos determinados unívocamente en función de una serie de variables independientes (el estado de un sistema depende de su historia previa, la inversión de comportamiento aleja irreversiblemente de la situación inicial, la inmutación no es vía de desgaste o destrucción, es el modo en que existe el hilemorfo como pieza singular en una trama de interdependencias e influencias-inflaciones exteriores transformantes por las que escapa a su propia corrupción, la ralentiza o la retrasa). La espontánea evolución teleotrópica procura mínima entropía y máxima entalpía al sistema que la experimenta (mínimo desorden y máxima enérgeia, menor alícuota de potencia y máxima carga ontológica de acto en el complejo acto-potencial por orientación real a un fin último netamente energético o actual) ${ }^{6}$. Una física errada que se desgarra en su centro.

La ruta dinamicista del quod est es indeterminista (Knuuttila, 1979: 76-80), el eîdos, matriz óntica, es indiferente en su realitas secundum quid de esencia a la existencia, por su indiferencia genética a la singularidad, signo ontológico último posibilitante de aquella. En esta diáspora metafísica que invierte la prioridad relativa de principios alzando la potencia en modo ontológico primario sobre el acto, la forma es efecto de causalidad eficiente o el cambio formal es resultado de la mutación de la materia, principio pasivo primordial, dotado de actualidad marginal, independiente de la forma, en el que están prefiguradas germinalmente (reedición matizada de la doctrina estoico-agustiniana de las rationes seminales) las formas que pueden ser inducidas o educidas por el poder efectivo del agente, precontenidas como actos virtuales, a su vez, en tal poder, de suerte que el cambio cualitativo es manifestación del cuantitativo.

6 "La anterioridad del acto y la potencia rige ahora en el sentido siguiente: la estructuración aislada no existe. La composición hilemórfica completamente ajena a un dinamismo energotélico es puramente potencial, es decir, no se da en la realidad actual. El compuesto hilemórfico es mera condición de posibilidad para el dinamismo energotélico y su realidad actual consiste en ser la composición hilemórfica «de» tal realidad interactuante e interdependiente con tal entorno. El mero compuesto hilemórfico carente de dinamismo, el cuerpo totalmente estático y aislado, el vacío energotélico no existe. Toda composición hilemórfica real, actual, está actualizada por el dinamismo energotélico de la que es a la vez causa y efecto (se trata de un feed-back o interacción). Todo compuesto hilemórfico está generado por un proceso genético y está expuesto a una continua modificación debida a la interacción dinámica del compuesto con su entorno. A su vez, todo dinamismo energotélico tiene un soporte hilemórfico que lo hace posible. Es una condición de posibilidad -potencia- del dinamismo energotélico (acto)" (Miralbell, 1994: 117-118). 
En el decadente friso energetista del Éxodo, la condición de creaturalidad descansa en la complejidad cum his onto-eidética (la criatura es pseudo-compuesto cum his de esencia y esse, por ser el actus essendi de orden extra-predicamental, extrínseco al plano de constitución sustancial), abonada la causalidad ejemplar a la esfera noética divina, supeditado su cometido a la voluntad ad extra. La criatura es esencialmente ex nihilo, su esencia no envuelve al esse, como sucede en el caso divino, ni atesora realidad alguna en sí misma al margen de esa composición; la criatura es esencialmente $a b$ alio, con el esse como factor de abaliedad-asimilación (esse tendría aquí valor de acto existencial, de suerte que la entidad finita «es esencialmente ab alio» porque su esencia es nada sin la recepción ab alio del esse como actus essentiae o acto de ser esenciado).

La emergencia ontológica de la potencia por anástrofe axiomática transforma el panorama de la finitud, depositando en la propia esencia una prístina razón de creaturalidad en forma de existibilidad: la esencia en su potencia real-metafísica reviste condición creatural, ya no radicada en la complejidad esencia-esse bajo restrictiva concepción analógica del esse como actus essendi. La dicotomización del esse (essentiae / existentiae) depara a la esencia un esse simpliciter essentiae, aval ontológico de intención o de creaturalidad real en potencia a la existencia. Articulan el nuevo paradigma dos aspectos del existible: ejemplaridad y contingencia. El primero evoca la realidad de la esencia, eslabón de causalidad ejemplar, revalorizando esta como auténtica causalidad, investida del halo de contingencia delator de incepción volitiva en toda variante de causalidad ad extra, aun ineficiente por no poner extra suam causam a su término, que no es efecto sino simple producto (la causalidad ejemplar, bajo mérito de tal, deberá tener un término quodammodo o secundum quid real, ese término es la esencia en su entidad metafísica). La mácula de contingencia ínsita en una ratio creaturalitatis transborda al dominio volitivo; en consecuencia, la ejemplaridad de la esencia, si ha de adverarse en ella el fruto de alguna variedad causal, no podría inscribirse, como pretende el tomismo, en la noosfera divina, antes bien, debe ser trasvasada al horizonte intencional del entendimiento práctico (Enrique de Gante: esse essentiae como prima intentio creationis) o al de la voluntad, motio contingens endógena que hace de la posibilidad lógica de ser (ordo idearum) una posibilidad real (ordo 
essentiarum), de la simple idea, una esencia, por ratificación ontológica con un esse essentiae (esse extra animam menor); del posible lógico, un existible, y la crasa existibilidad que declara esencialmente en sí el futuro contingente (si Dios es esencialmente existente, la criatura es esencialmente existible) es ya divisa de la creaturalidad de que adolece la idea, la mera existibilidad es indicador de realidad esencial o metafísica (infra-existencial), inactual, no de realidad simpliciter individuada (Duns Escoto). En esa moción volitiva interna, Dios reconoce uno de los extremos de la alternativa como creable, antes de su efectuación, y esa dilección ineficaz aún cae ya bajo dominio potestativo de la voluntas ad intra, representa una intención de creación, no una simple idea de creación (estatuto pre-creatural), en la cual halla certificación ontológica de esencia un creable (no sencillamente inteligible en su compacta arquitectura o raquis formal).

La realidad metafísica de la esencia enlaza en su potencia objetiva con la posición ejemplar, pues el creable ya no es solo una idea en el entendimiento especulativo divino, un simple esse intelligibile en su posibilidad lógica de ser, sino una intención en el orden práctico o un esse volibile en acto virtual en la potencia volitiva-eficiente del creador. Esa base de ejemplaridad traduce una intención arraigada en la voluntad interna divina que servirá de ratitudine ontológica al acto eficiente de expatriación causal, pero en el singular ya existenciado (voluntad efectiva divina) tiene como expresión vestigial la realidad secundum quid de una esencia, indeterminada, neuter ex se en su unitas naturae infranumérica, fondo de realidad metafísica (sub-existencial) común (comunidad real eidética en la unidad infranumérica, no en la unidad supranumérica de universalidad o comunión lógico-predicativa) a los individuos que la actualizan. Obviamente, la esencia no subsiste en su realidad disminuida separada del singular existente (un espacio ejemplar de esencias subsistentes al modo del kósmos noetós platónico), es solo una dimensión eidética (real) de lo actual de facto, distinta de su realidad simpliciter física-individuada e indiferente a ella, pero solo existente en singular modalización. La causalidad ejemplar es ineficaz, no tiene efecto sustraído a la fáctica acción formalizadora, pero sí rédito propio: la dimensión de realidad quiditativa subyacente a la física-existencial singular que prodiga comunidad real en naturaleza a la constelación de individualidades que la encarnan, comunidad real por cuanto rubrica un nexo real con Dios ejemplar (no un vínculo puramente ideal). La 
física cuántica sintoniza con la espiral de existibilidad (condición de realidad de la nuda potencia o posibilidad efectiva de ser): un sistema cuántico es, en esencia, una colección de estados cuantizados de existibilidad que anuncian colapsos actuales de la función de onda.

La estratificación formal de la entidad, la pluralidad de formas sustanciales concertadas en unidad entitativa, brinda ocasión causal a los agentes naturales en la causación descendente, en detrimento de la abusiva solución de unicidad formal (unum esse est ab una forma) que desvirtúa la causalidad intermedia. En la novedosa metafísica, el fin languidece, se devalúa, desvaído su vigor causal (real): el fin es epifenoménico, mueve metafóricamente, subordinado a la eficiencia, sin la cual es puramente potencial, una posibilidad irrealizada pendiente de la acción eficiente (solo es actual en cuanto actualizado por la eficiencia que opera bajo su inspiración o por amor a él). Con la tímida guía metafórica del fin palidece la teleonomía ${ }^{7}$ (telotaxias, teleotropismos...), contraída, por anterioridad de la eficiencia, a sus formas teleológicas intencional o supra-intencional. El imago que persigue la metamorfosis incompleta de la ninfa es un potencial estadio final, sin realidad nítida hasta la última ecdisis, solo como imagen a consumar preside el hemimetabolismo de la inmadura libélula en una teleonomía metafórica. La incardinación acto-potencia que el aristotelismo delega en la díada causal fin-eficiencia se ha alterado, la potencia recae ahora sobre el fin, solo actual en cuanto el auténtico poder causal, el eficiente, se adhiere a él de facto en su acción. Y la potencia preponderante es la facultad volitiva, genuina potencia libre en el modus eliciendi, no natural o determinada ad unum, indeterminada ex se por autosuficiencia activa-superabundantis sufficientiae, quae est ex illimitatione actualitatis, simpliciter (voluntad divina) vel quodammodo (voluntad creatural)-, la materia pre-forme lo estaría por insuficiencia activa (indeterminatio insufficientiae sive ex potentialitate et defectu actualitatis) (Duns Escoto, 1891/95, IX, q. 15: 608-610):

\footnotetext{
${ }^{7}$ Connivencia con Monod: el vocablo designará las formas infra-intencionales de teleología en su calidad de aparente propósito y ordenación a objetivos estructurales y funcionales de los seres animados. "L'objectivité cependant nous oblige à reconnaître le caractère téléonomique des êtres vivants, à admettre que dans leurs structures et performances, ils réalisent et poursuivent un projet. Il y a donc là, au moins en apparence, une contradiction épistémologique profonde. Le problème central de la biologie, c'est cette contradiction elle-même, qu'il s'agit de résoudre si elle n'est qu'apparente, ou de prouver radicalement insoluble si en vérité il en est bien ainsi” (Monod, 1970: 37-38).
}

SCIO. Revista de Filosofí, n. ${ }^{\circ}$ 19, Noviembre de 2020, 209-228, ISSN: 1887-9853 
materia y voluntad se perfilan como instancias contrapuestas (no tanto antagónicas) en su carácter indeterminado, la esencial potentia contradictionis pasiva de la materia frente a la autoactiva potentia ad opposita de la voluntad, en cuya prestigiada potestad subsumen a simultaneo los opuestos. La materia se ofrece limitadamente en su pasividad al agente natural, por detentar este una potencia limitada (ad unum), pero se somete ilimitadamente en su potencialidad a la ilimitación activa (simpliciter / quodammodo) de la potencia libre, en ambos casos, la limitación o ilimitación de la sumisión se referirá al espectro de formas recibidas, al modo de recepción y al orden de sucesión de estas $^{8}$. La inédita metafísica de la posibilidad pura está en marcha, preludio del indeterminismo físico.

\section{§4. ConClusión}

La metafísica del quod est, por el acta ontológica de la potencia en tesitura de esencia creable, transforma la concepción de la realidad y la creaturalidad: prima ratio creaturalitatis apunta al esse essentiae, un ser ontológicamente ratificador de esencia, en la que estarían codificados, en clave de posibilidad real (acto virtual), todos los valores propios o individuantes que la cuantizarán en acto incomunicable ut quod (colapsos singulares de una función de onda que encubre el eîdos). La razón versal de creaturalidad incoa un expediente esencial ligado a la causalidad ejemplar y subyacente a la dependencia actual ab alio que dicta una creaturalidad efectiva asociada a la causalidad eficiente. La simple condición de creable o existible entraña ya un índice de realidad, no simpliciter, solo secundum quid, la realidad de la posibilidad existencial en sí misma habilitada en la esencia como entidad ontológicamente ratificada,

8 "Limitate subditur agenti, quantum scilicet primo ad formas determinatas. Secundo, quantum ad modum determinatum recipiendi illas ab ipso. Et tertio quantum ad ordinem determinatum recipiendi hanc post illam [...] Sed quia ordo ille non est necessarius, nisi in quantum materia transmutatur ab agente naturali, cuius virtuti non subiicitur ille ordo [...] hic habet locum potentia obedientialis, secundum quam materia cuiuscumque formae capax est immediate post quamcumque, per transmutationem $\mathrm{ab}$ agente, cuius virtuti subest dictus ordo [...] vel illimitate subditur, scilicet quantum ad receptionem cuiuscumque perfectibilis inesse, et quocumque modo et quocumque ordine" (Duns Escoto, 1891/95, IX, q. 12: 578) 
más allá de la nuda posibilidad lógica de ser por íntima coherencia formal. La esencia embosca una "nube" de probabilidades individuales, eclosionando en existencia cuantizada (las singularidades son modos virtuales internos de proyección actual de una esencia, real justamente como fondo metafísico común a las individualidades en que cristaliza). La razón de creaturalidad deja de radicar en la composición cum his esse-esencia amparada en la causalidad eficiente (eje del quo est) para consolidar en la esencial existibilidad o aptitud existencial connotada por la indiferencia a la existencia de una esencia prendida en su realidad infra-existencial a la causalidad ejemplar (meridiano del quod est), e individualizable sin excepción por ser indifferens de se ad singularitatem (no de se haec) y por ser la individualidad modus intrinsecus essentiae, ya no estigma de complejidad ex his hilemórfica para la esencia (la subsistencia mórfica no admite pluralidad individual).

Si la función de onda no reviste en sí misma un significado físico convencional, no es evaluable como una función clásica de onda electromagnética puesto que sus valores propios no son necesariamente reales, precisando un reajuste de significado físico que manejaría la densidad de probabilidad de localización de una partícula en el entorno de un nodo (producto de la función de onda por la compleja conjugada $)^{9}$, tampoco la esencia es simpliciter real en tanto que esencia, su consistencia como quididad (en la unitas naturae característica) no es física (esa unitas no es universal ni numeral, sino infranumérica). Aunque su existencia sea singular, su realidad como esencia es de otra índole, no fáctica-existencial (función de onda colapsada en un valor propio), la del evento contingente actual, sino la de su misma posibilidad, solo efectuada en singular modalidad. Los estados posibles encriptados en la función de onda, los valores discretos de energía que cuantizan la realidad microscópica, son afines a la visión de la esencia como posibilidad real pura de un discontinuo de singularidades que la actualizan, trasfondo real-metafísico (realidad secundum quid) a esas contingencias físicas actuales (realidad simpliciter).

\footnotetext{
${ }^{9}$ La densidad de probabilidad (cuadrado de la función de onda de la partícula descrita por la ecuación de Schrödinger) o la probabilidad de localización puntual de una partícula por unidad de volumen será proporcional al tamaño de este elemento $(d V)$ y genéricamente dependiente del tiempo y la posición. Para ondas estacionarias, la densidad de probabilidad es independiente del tiempo. Todo número complejo es susceptible de modulación por simple multiplicación por el conjugado.
} 
La mecánica cuántica es afín al indeterminismo metafísico del quod est y la consecuente sintaxis de mundos posibles, a la lógica de modalidades que escruta la posibilidad real de ser (no una simple posibilidad lógica sino ontológica, valores energéticos discretos que se postulan como intrínsecas alternativas probables de singularidad física) e instaura la contingencia sincrónica, la metafísica del salto cuántico al plano quiditativo del esse possibile desde el factum contingente. El colapso ontológico singular de la esencia en "energética" (actual) cuantización y la discontinuidad existencial (realitas simpliciter) de un fondo de realidad metafísica (realitas secundum quid) comunísimasupragenérica (ens univocum) o común-específica (natura communis) son el introito, la cadencia preliminar a la mecánica de quanta. La razón devuelve las abstracciones a su realidad extrapsíquica sin desistir de la inquisición de principios primeros o predicamentales. Agotada su competencia en el hallazgo de una unidad concausal del ser, resta la residual distinción de esencia y existencia. El ejercicio apofático de la ontología modal por olvido de los principios próximos la oblitera al margen de la existencia, invitando al abandono de las nociones transcendentales, sustituidas por modales, terreno cultivado para el positivismo.

El férreo determinismo energotélico del Éxodo cede el relevo al indeterminismo. La materia, causa intrínseca primaria, goza de realidad creatural propia, quantum ad fieri et ad esse, marginada de su sociedad con la forma, un crisol mórfico con esse primum quasi-informe (en ella están inseminadas las formas sustanciales que la actualizarán por donación de esse simpliciter). La siembra mórfica es motivo detonante, la forma pura es acto virtual-ratio ejemplar en el poder activo o ratio seminal expectante en la materia (precontención virtual de la forma en la potencia del agente y germinal en la pasividad hilética). Las causas extrínsecas se actualizan como tales causas en la medida en que despliegan su función hilemorfizadora, su realidad es activa, silentes son solo potenciales. Aun cuando el poder activo sea anterior a sus efectos, virtualmente prefigurados en él (la forma como ratio exemplar o causa formal extrínseca, la forma a priori, sin materia, previa a su efectuación en esta, es virtualmente actual en el poder eficiente), de suyo no es sino el poder de causar lo efectuable, lo formalizable-materializable (hilemorfismo universal), y este enfoque de la causalidad extrínseca traba la viabilidad de 
conclusiones necesarias a partir de premisas referidas a seres contingentes, quebrando el trazado netamente quia de las vías teológicas por inflexión $a$ priori, metábasis de la evidencia del hecho contingente (existencia de seres efectuados, finalizados o excedidos en perfección) a su esencial existibilidad (plano quiditativo, reformulación de proposiciones contingentes en términos de possibile: esencial posibilidad de lo efectuable, finalizable o excedible en perfección).

El axioma de prioridad crono-ontológica del acto sobre la potencia se ha truncado. La hegemonía inter- e intraentitativa del acto que privilegiara, en el orden transcendental en que el acto es esse, al esse subsistens sobre el compuesto onto-eidético, y aun dentro de este, al esse creaturae (prima rerum creatarum) sobre la esencia, es subvertida: preeminencia de la potencia (extrapolada a la imagen de Dios, no tanto esse cuanto potestas). Y si el acto cotiza transcendentalmente como esse, la potencia lo hará como esencia, derrocando al acto en primado metafísico por credencial ontológica de existibilidad, latente en el singular actual, interlocutor con Dios-ejemplar. La función cuadrática de onda puntea posibilidades, probabilidades regionales de presencia, partícipe de una ecuación cuyas soluciones, cuantizadas, censan posibles estados ontológicos de un sistema. La diáspora del quod est celebra (contra la metafísica del Éxodo) la regencia de la insurgente potencia frente a la tiránica soberanía del acto, y esa axial indeterminista se alinea con el orden cuántico, comulgando con el espíritu que lo impulsa: la posibilidad en indumento fenomenológico antes que el suceso.

REFERENCIAS BIBLIOGRÁFICAS

Bardi, J. S. (2006). The Calculus Wars. The Greatest Mathematical Clash of All Time. New York: Thunder's Mouth Press.

Cohen, B. (1989). El nacimiento de una nueva Física. Madrid: Alianza Universidad.

De Garay, J. (1987). Los sentidos de la forma en Aristóteles. Pamplona: Eunsa.

Duns Escoto, J. (1891/95). Quaestiones subtilissimae super libros Metaphysicorum Aristotelis. Paris: Vivès VII.

SCIO. Revista de Filosofia, n. ${ }^{\circ}$ 19, Noviembre de 2020, 209-228, ISSN: 1887-9853 
Evans, L. C. (1998). Partial Differential Equations. Graduate Studies in Mathematics, 19. Providence: American Mathematical Society.

Faust, A. (1931). Die Möglichkeits Gedanke. Systemgeschichtliche Untersuchungen. Heidelberg: Carl Winters Universitätsbuchhandlung.

Gamow, G. (1971). Biografía de la física. Barcelona: Salvat.

Hoeres, W. (1976). La volontà como perfezione pura in Duns Scoto. Padova: Liviana Editrice.

Kielkopf, CH. F. (1978). Duns Scotus's Rejection of 'Necessarily Exists' as a Predicate. Journal of History of Philosophy (16/1), 13-21.

Knuuttila, S. (1976). Determinismi keskiajan aristotelismin ongelmana. Teologinen Aikakauskirja (79), 434-449.

Knuuttila, S. (1976). Duns Scotus ja mahdollisuuden 'statistisen' tulkinnankritiikki (Duns Scotus' Criticism of the 'Statistical' Interpretation of Possibility). Reports from the Institute of Philosophy, University of Helsinki.

Knuuttila, S. (1978). The Statistical Interpretation of Modality in Averroes and Thomas Aquinas. Ajatus (37), 79-98.

Knuuttila, S. (1979). The Change of Modal Paradigms in Late Medieval Philosophy. Abstracts of the 6th International Congress of Logic, Methodology and Philosophy of Science, Sect. 13-14, Hannover, 76-80.

Messiah, A. (1999). Quantum Mechanics. New York: Dover Publications.

Miralbell, I. (1994). El dinamicismo voluntarista de Duns Escoto. Una transformación del aristotelismo. Pamplona: Eunsa.

Monod, J. (1970). Le hasard et la nécessité. Paris: Éditions du Seuil.

Pape, I. (1966). Tradition und Transformation der Modalität I: MöglichkeitUnmöglichkeit. Hamburg: Felix Meiner Verlag.

Tipler, P. A., Mosca, G. (2012). Física moderna. Mecánica cuántica, relatividad y estructura de la materia. Barcelona: Reverté.

Westfall, R. S. (1980). La construcción de la ciencia moderna. Barcelona: Labor. 